\title{
Statistik der Benutzung 2011
}

Im vergangenen Jahr sind die meisten Kennzahlen der Benutzung im Vergleich zum Vorjahr auf hohem Niveau stabil geblieben. Erfreulicherweise haben wir nach dem Einbruch in 2010, der wohl hauptsächlich auf die Einführung der Benutzungsgebühren zurückzuführen ist, wieder einen leichten Aufwärtstrend sowohl bei den aktiven als auch den neuen Benutzern zu verzeichnen. Den größten Zuwachs registrierten wir im Bereich der Information, konkret bei den schriftlichen Anfragen, den Führungen und Schulungen, sowie in der aktiven und passiven Fernleihe bei der Bearbeitung elektronischer Bestellungen.

\begin{tabular}{|c|c|c|c|c|c|}
\hline \multirow[b]{2}{*}{ Allgemeine Angaben zur Benutzung } & \multirow[t]{2}{*}{2009} & \multicolumn{2}{|c|}{2010} & \multicolumn{2}{|c|}{2011} \\
\hline & & & $\%$ & & $\%$ \\
\hline Aktive Benutzer (nur Jahresstatistik) & 36.949 & 32.833 & $-11,1$ & 33.098 & $+0,8$ \\
\hline Neue Benutzerausweise & 10.719 & 10.649 & $-0,7$ & 10.857 & $+2,0$ \\
\hline Öffnungstage & 289 & 292 & $+1,0$ & 293 & $+0,3$ \\
\hline Entleihungen insg. (EDV/konv.: DBS) & 1.366 .892 & 1.327 .607 & $-2,9$ & 1.311 .111 & $-1,2$ \\
\hline Vormerkungen (DBS) & 203.820 & 185.982 & $-8,8$ & 185.482 & $-0,3$ \\
\hline \multicolumn{6}{|l|}{ Information } \\
\hline Auskunftsanfragen (DBS) & 32.743 & 29.550 & $-9,8$ & 30.256 & $+2,4$ \\
\hline \multicolumn{6}{|l|}{ davon schriftl. Anfragen (Kopieraufträge, } \\
\hline Briefe, E-Mails, Anschaffungsvorschläge) & 1.105 & 922 & $-16,6$ & 1.095 & $+18,8$ \\
\hline Schulungen + Führungen / Teilnehmer & 2.763 & 2.425 & $-12,2$ & 2.767 & $+14,1$ \\
\hline \multicolumn{6}{|l|}{ Hauptlesesaal } \\
\hline Benutzungen (Teilungsfaktor 2) & 299.902 & 292.128 & $-2,6$ & 298.722 & $+2,3$ \\
\hline \multicolumn{6}{|l|}{ Fernleihe (aktiv u. passiv) } \\
\hline Bestellungen anderer Bibliotheken & 89.929 & 91.139 & $+1,3$ & 81.991 & $-10,0$ \\
\hline elektronischer Versand (Scans) & & 1.947 & & 4.565 & $+134,5$ \\
\hline Eigene Bestellungen (Pass. Leihverkehr) & 28.846 & 25.673 & $-11,0$ & 24.156 & $-5,9$ \\
\hline erhaltene Scans & & 288 & & 1.140 & $+295,8$ \\
\hline
\end{tabular}

Bemerkenswert ist unsere Benutzerstruktur: nach wie vor sind gut über die Hälfte Studierende, ca. ein Viertel sind Schüler sowie sonstige Auszubildende und nur ca. ein Viertel sind Berufstätige was sich natürlich in der Einnahmesumme der Benutzungsgebühren widerspiegelt. Aber auch ohne die Zusammensetzung unserer Klientel zu kennen, würden wir diese doch erahnen können...vor allem aufgrund der in den vergangenen Wochen im Haus vorherrschenden "Bienenkorbatmosphäre". 